\title{
Recent results from the COMPASS hadron program
}

\author{
Sebastian Uhl ${ }^{1, a, b}$, on behalf of the COMPASS Collaboration \\ ${ }^{1}$ Technische Universität München, James-Franck-Straße, 85748 Garching
}

\begin{abstract}
COMPASS is a fixed-target experiment aimed to study the structure and dynamics of hadrons. Using a $190 \mathrm{GeV} / c$ pion beam, the spectrum of light mesons is studied at four-momentum transfers squared to the target between 0.1 and $1.0 \mathrm{GeV}^{2} / c^{2}$. The flagship channel is the diffractive production of $\pi^{-} \pi^{-} \pi^{+}$final states for which COMPASS has recorded the world's largest data sample. The determination of properties of known resonances, and the search for new states is also pursued in the $\pi^{-} \pi^{0} \pi^{0}$ final state, and in centrally produced systems. The structure of light mesons is studied in Primakoff reactions at lowest four-momentum transfers. Using these processes, the polarizability of the pion, the radiative width of the $a_{2}(1320)$ and, for the first time, that of the $\pi_{2}(1670)$ have been measured.
\end{abstract}

\section{Introduction}

The Common Muon and Proton Apparatus for Structure and Spectroscopy (COMPASS) is a fixedtarget experiment located at CERN's Super Proton Synchrotron (SPS). It is aimed to study the structure and dynamics of hadrons. Spectrum and structure of light mesons are investigated using reactions of hadron beams with different targets. The spectrum of light mesons is studied in two different processes. Isovector states are diffractively produced using a pion beam impinging on a liquid-hydrogen target (figure 1(a)), isoscalar states are produced in central production reactions of a proton beam also with a liquid-hydrogen target (figure 1(b)). The structure of light mesons is investigated in Primakoff reactions of a beam pion and a heavy (high $Z$ ) target (figure 1(c)). The decay products of the intermediate states are detected in a two-stage magnetic spectrometer equipped with an electromagnetic and hadronic calorimeter in each spectrometer stage [1]. This provides full coverage for charged and neutral particles, resulting in a homogenous acceptance over a rather wide kinematic range.

\section{Diffractive Dissociation into three Pions}

Concerning diffractive production of intermediate states, the diffractive dissociation of a beam pion into the three-pion final state is the dominant process. In COMPASS it can be studied in two different channels simultaneously, namely in $\pi^{-} \pi^{-} \pi^{+}$and $\pi^{-} \pi^{0} \pi^{0}$, providing an immediate handle on systematic

\footnotetext{
ae-mail: sebastian.uhl@mytum.de

${ }^{b}$ The author acknowledges financial support by the German Bundesministerium für Bildung und Forschung (BMBF), by the Maier-Leibnitz- Laboratorium der Universität und der Technischen Universität München, and by the DFG Cluster of Excellence "Origin and Structure of the Universe" (Exc153).
} 


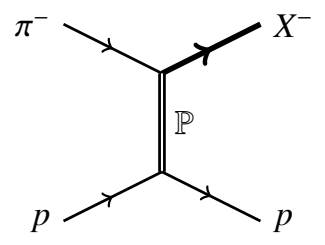

(a) diffractive dissociation

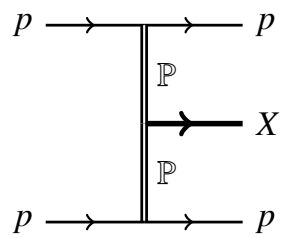

(b) central production

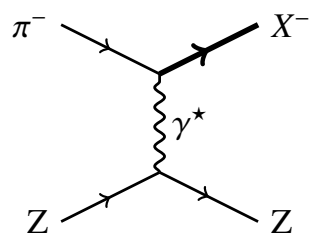

(c) Primakoff reaction

Figure 1. The different production mechanisms used for the present studies.

effects, as the reconstruction of relies on different parts of the detector. About 50 million exclusive $\pi^{-} p \rightarrow \pi^{-} \pi^{-} \pi^{+} p$ events in a range of the four-momentum transfer squared $t^{\prime}$ from the beam to the target between 0.1 and $1.0 \mathrm{GeV}^{2} / c^{2}$ have been recorded with a pion beam impinging on a liquidhydrogen target. The same data set contains roughly 3.5 million events with $\pi^{-} \pi^{0} \pi^{0}$ in the final state.

Finally, the data are decomposed into spin-parity components using a partial-wave analysis employing the isobar model. Figure 2 shows the process under study. The incoming beam pion diffractively scatters off a proton in the liquid-hydrogen target producing an intermediate state $X^{-}$, which subsequently decays into a two-pion isobar $R_{\pi \pi}$ and a bachelor pion with a relative orbital angular momentum $L$ in between. The isobar decays further into two pions. For the present analysis $\rho(770)$, $f_{0}(980), f_{2}(1270), f_{0}(1500), \rho_{3}(1690)$, and a broad $(\pi \pi)_{S}$ component have been used. The applied fit model contains partial waves with the spin $J$ of $X^{-}$and the angular momentum $L$ between the isobar $R_{\pi \pi}$ and the bachelor pion going up to 6 . Out of the possible combinations, 87 waves with non-negligible intensity are kept for the final analysis: 80 with a positive reflectivity $\varepsilon=+1$ and 7 with $\varepsilon=-1$. In addition one incoherent wave with an isotropic angular distribution is included.

The process is factorized into one part describing the production of the state $X^{-}$, and a second one describing its decay. The latter part can be calculated from the five phase-space variables given the quantum numbers of the state $X^{-}$and the decay channel. The production amplitude are extracted by a fit, which maximizes the likelihood of observing the data given the model taking into account the detector acceptance.

The three-pion mass range between 500 and $2500 \mathrm{MeV} / c^{2}$ is studied in bins of $20 \mathrm{MeV} / c^{2}$ for the $\pi^{-} \pi^{-} \pi^{+}$channel and $40 \mathrm{MeV} / c^{2}$ for the $\pi^{-} \pi^{0} \pi^{0}$ channel. The data are divided into eleven bins of

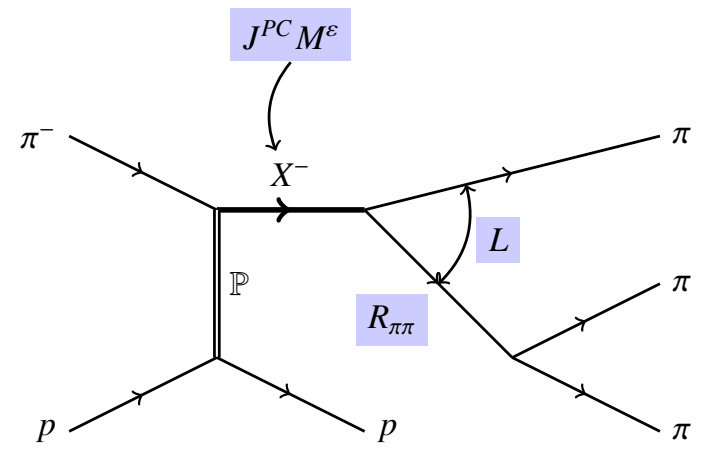

Figure 2. Sketch of the process under study. 


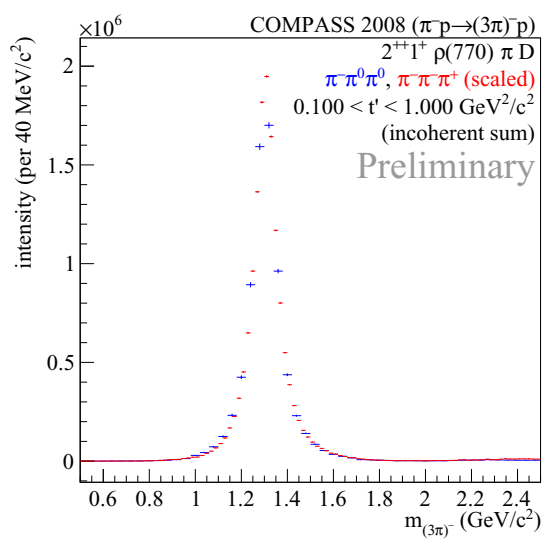

(a) $2^{++} 1^{+} \rho(770) \pi D$

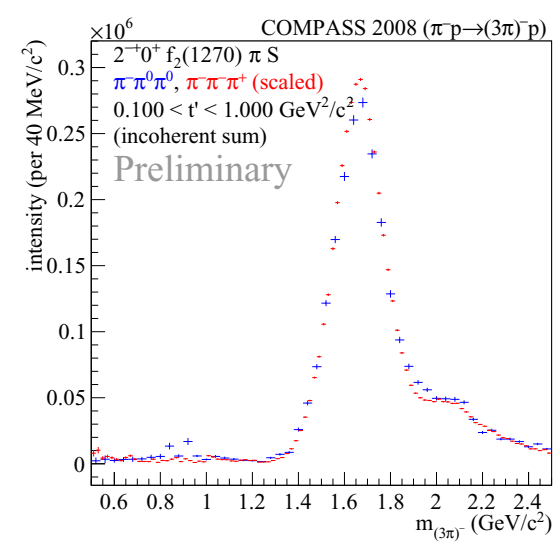

(b) $2^{-+} 0^{+} f_{2}(1270) \pi S$

Figure 3. Sum of partial-wave intensities over all $t^{\prime}$ bins for two of the waves with a high intensity.

$t^{\prime}$ for the $\pi^{-} \pi^{-} \pi^{+}$channel (eight bins for $\pi^{-} \pi^{0} \pi^{0}$ ), such that the number of events over the analyzed mass range is approximately equal for all the bins. A rank-1 fit in each of those bins is performed. Figure 3 shows the intensity sum over all $t^{\prime}$ bins for two high-intensity waves. The wave names $J^{P C} M^{\varepsilon}$ isobar $\pi L$ contain the quantum numbers $J^{P C} M^{\varepsilon}$ of the intermediate state $X^{-}$and information on the decay channel. The $2^{++} 1^{+} \rho(770) \pi D$ wave with the dominant $a_{2}(1320)$ is shown in figure 3(a), the $2^{-+} 0^{+} f_{2}$ (1270) $\pi S$ wave with the $\pi_{2}$ (1670) and a tail towards higher masses in figure 3(b). In both cases the results for the $\pi^{-} \pi^{-} \pi^{+}$(red markers) and the $\pi^{-} \pi^{0} \pi^{0}$ (blue markers) channel are overlaid and in good agreement. Here and in the following plots the two channels are normalized on the integral for each plot individually. For those two waves, the shape of the intensity spectra does not change with $t^{\prime}$. This is in contrast to the $1^{++} 0^{+} \rho(770) \pi S$ wave shown in figure 4 . For this wave, in which a strong $a_{1}(1260)$ is expected, the peak of the intensity shifts by about $100 \mathrm{MeV} / c^{2}$ over the studied $t^{\prime}$ range indicating a rather large non-resonant contribution.

Apart from these three largest waves, also for smaller waves good agreement for the two channels is found. One particularly interesting signal is found in the $1^{++} 0^{+} f_{0}(980) \pi P$ wave (see figure 5(a)). A narrow structure around $1400 \mathrm{MeV} / \mathrm{c}^{2}$ is observed in both channels. In addition to the intensity peak, also a rapid phase motion with respect to other waves is found. This previously unobserved signal could correspond to a new state $a_{1}(1420)$. Different parameterizations for the isobars, in particular for the $f_{0}(980)$ and the broad $(\pi \pi)_{S}$ component, have been tested to exclude a possible artifact from the used model.

Finally, resonance parameters are extracted from a fit of Breit-Wigner amplitudes to a subset of the spin-density matrix for the $\pi^{-} \pi^{-} \pi^{+}$channel. Apart from the intensities, this fit also takes into account the interferences between the waves. In addition to the four waves shown above, the $0^{-+} 0^{+} f_{0}(980) \pi S$ and $4^{++} 1^{+} \rho(770) \pi G$ waves are used. The model includes Breit-Wigner functions for the $a_{1}(1260)$, an $a_{1}^{\prime}$, the $a_{2}(1320)$, an $a_{2}^{\prime}$, the $\pi_{2}(1670), \pi_{2}(1880), \pi(1800), a_{4}(2040)$, and for the new $a_{1}(1420)$. Also a non-resonant term is included for each wave. The Breit-Wigner parameters are obtained from a simultaneous $\chi^{2}$ fit to the spin-density submatrix of the six waves in all eleven $t^{\prime}$ bins.

The extracted parameters of the major resonances are in agreement with previous measurements by COMPASS and other experiments [2]. Figure 5(b) shows the result from this fit for the new $a_{1}(1420)$. The intensity extracted by the spin-parity decomposition (black markers) is overlaid with 
EPJ Web of Conferences

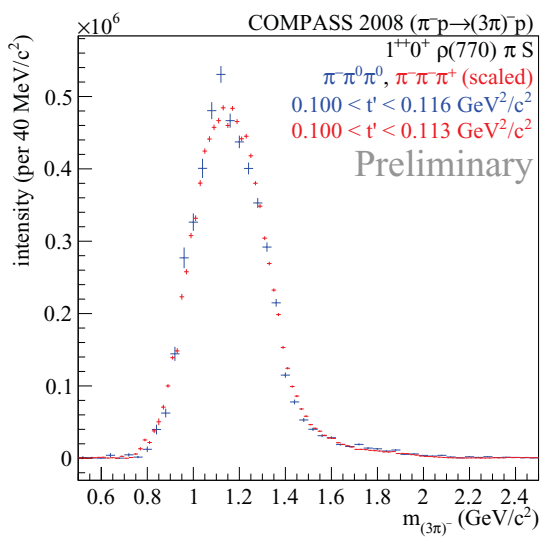

(a) low $t^{\prime}$

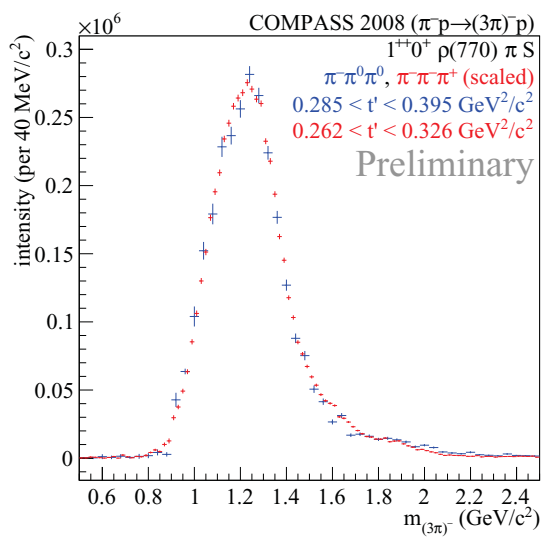

(b) high $t^{\prime}$

Figure 4. Partial-wave intensity of the $1^{++} 0^{+} \rho(770) \pi S$ wave in two $t^{\prime}$ regions.

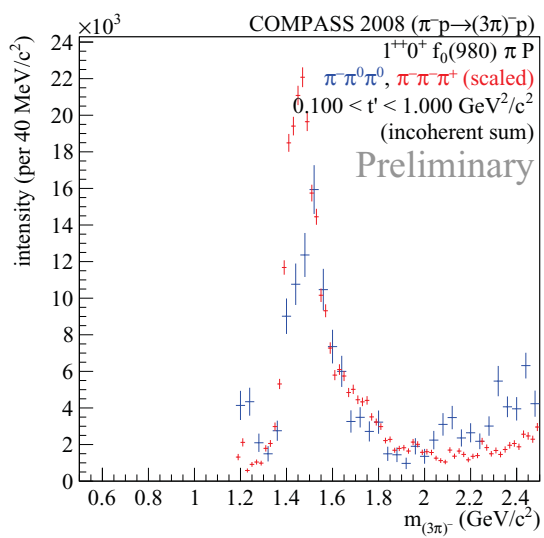

(a) intensity sum over all $t^{\prime}$ bins

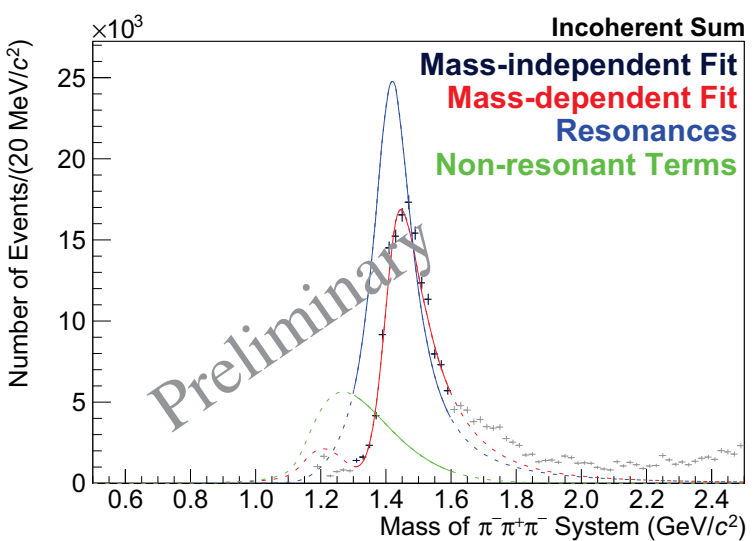

(b) fit to spin-density matrix

Figure 5. Partial-wave intensities of the $1^{++} 0^{+} f_{0}(980) \pi P$ wave.

the total result (red line) which is the coherent sum of a resonant (blue line) and a non-resonant (green line) contribution. A well determined mass of $M=1412-1422 \mathrm{MeV} / c^{2}$ and a narrow width of $\Gamma=130-150 \mathrm{MeV} / c^{2}$ is obtained. This is in contrast to other signals of similar magnitude like the $a_{1}^{\prime}$, the $a_{2}^{\prime}$ or the $\pi_{2}$ (1880) for which a larger uncertainty in mass and width is retrieved from the same fit.

\section{Central Production}

The spin-parity decomposition cannot only be applied in diffractively produced systems, but also in centrally produced ones, e.g. in the reaction $p p \rightarrow p K^{-} K^{+} p$ of a proton beam impinging on a liquid- 

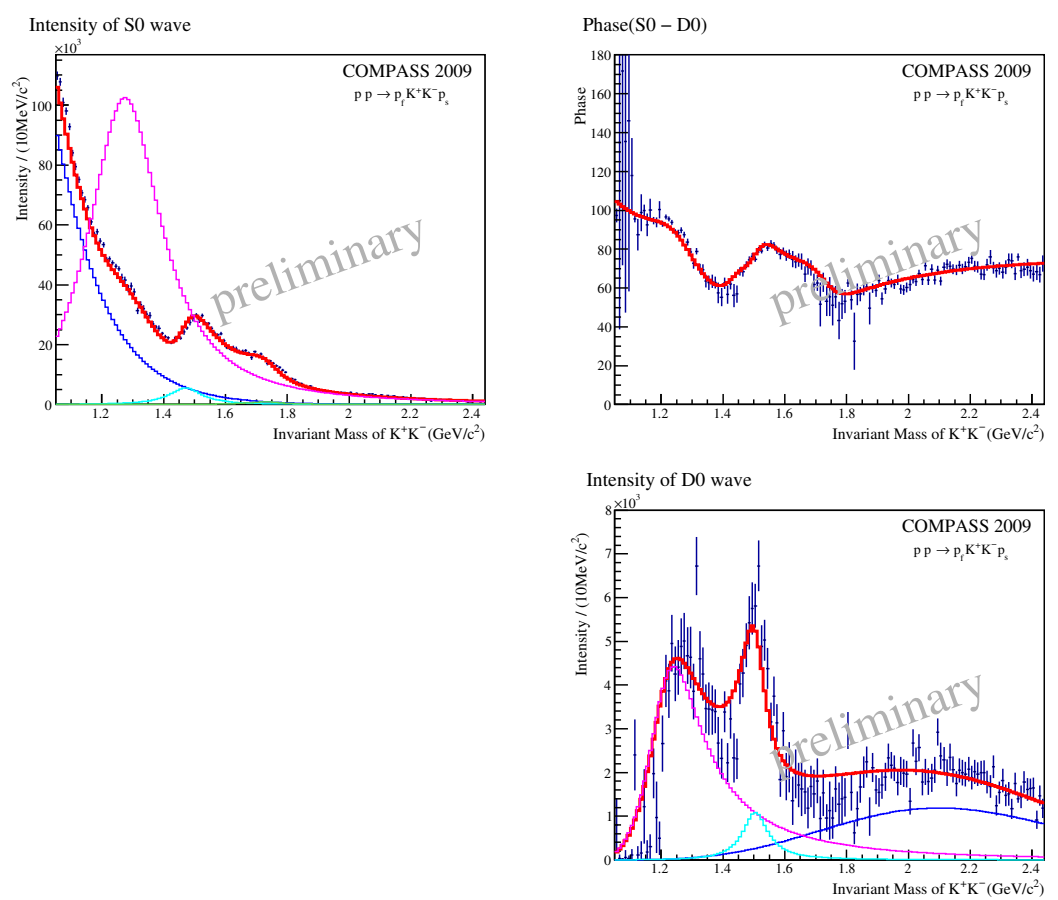

Figure 6. Spin-density matrix for the centrally produced $K^{-} K^{+}$system.

hydrogen target [3]. Kaons are identified in a ring-imaging Cherenkov detector. Centrally produced systems are selected by requiring a rapidity gap between the fast proton and the $K^{-} K^{+}$system.

The spin-parity decomposition is performed with four partial-waves, of which two collect negligible intensity, so that only the $S_{0}$ and $D_{0}$ waves are used to extract Breit-Wigner resonance parameters. Figure 6 shows the intensities of the two waves, and the phase between them (blue markers). Again, the mass dependence is described by Breit-Wigner amplitudes and a coherent background for each wave. The total result is shown by the red, the coherent non-resonant terms by the blue lines. Resonant contributions are shown in magenta, turquoise, and green. The $D_{0}$ wave is described using the $f_{2}(1270)$ and $f_{2}^{\prime}(1525)$. For the $S_{0}$ wave in addition to the $f_{0}(1500)$ and $f_{0}(1710)$, a $f_{0}(1370)$ is required in particular to get a good description of the phase around $1350 \mathrm{MeV} / c^{2}$.

\section{Radiative Widths}

Radiative decays $X^{-} \rightarrow \pi^{-} \gamma$ of resonances allow to measure electromagnetic transitions. Experimentally a direct measurement is challenging. The inverse process, that is the scattering of a pion off a Coulomb field producing an intermediate state $X^{-}$(see figure 1(c)) is called a Primakoff reaction. The Coulomb potential of a heavy nucleus acts as a source of quasi-real photons. The cross section for Primakoff production of a resonance is proportional to the width of its radiative decay. Since the exchange particle is a quasi-real photon, Primakoff produced states predominantly have spin projection $M=1$. Those states can also be produced diffractively, but the cross section for diffractively produced 


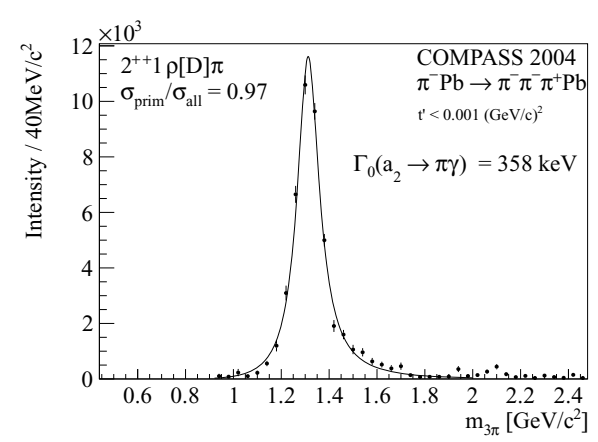

(a) $a_{2}(1320)$

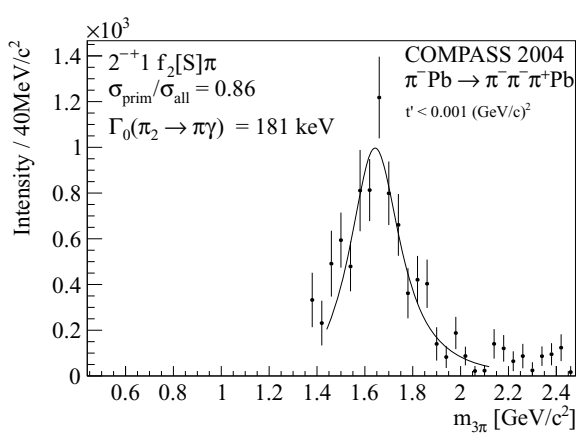

(b) $\pi_{2}(1670)$

Figure 7. Partial-wave intensities for the two waves used to extract the radiative widths.

states is proportional to $t^{\prime M \mid} e^{-b t^{\prime}}$, so that at small $t^{\prime} M=1$ states should predominantly be produced by the Primakoff reaction.

COMPASS has studied this reaction by scattering a pion beam off a thin disk of lead [4]. A partial-wave analysis is employed in order to decompose the collected data into spin-parity states, thereby also identifying the $M=1$ final states. Two waves have been considered to extract radiative widths, the $2^{++} 1 \rho(770) \pi D$ wave for that of the $a_{2}(1320)$, and the $2^{-+} 1 f_{2}(1270) \pi S$ wave for that of the $\pi_{2}(1670)$. At four-momentum transfers squared $t^{\prime}<0.001 \mathrm{GeV}^{2} / c^{2}$ the Primakoff production contributes $97 \%(86 \%)$ of the intensity found in the $2^{++} 1 \rho(770) \pi D$ wave (in the $2^{-+} 1 f_{2}(1270) \pi S$ wave, respectively). Note that in this case the intensities of the two different reflectivities $\varepsilon= \pm 1$ have to be summed up incoherently, as the production plane at such small $t^{\prime}$ is poorly measured due to limited experimental resolution. For both resonances the observed decay channels, $\rho \pi$ for the $a_{2}(1320)$ and $f_{2} \pi$ for the $\pi_{2}(1670)$, are not the only possible decay channels. This is taken into account by considering the relevant branching fractions.

A radiative width of the $a_{2}(1320)$ of $\Gamma_{0}\left(a_{2}(1320) \rightarrow \pi \gamma\right)=(358 \pm 6 \pm 42) \mathrm{keV}$ is measured, consistent with calculations from the VMD model [5]. For the first time a radiative width of the $\pi_{2}(1670)$ of $\Gamma_{0}\left(\pi_{2}(1670) \rightarrow \pi \gamma\right)=(181 \pm 11 \pm 27) \mathrm{keV}$ is measured.

\section{Pion Polarizability}

Polarizabilities describe the rigidity of a particle against deformations in an external electric $(\alpha)$ or magnetic $(\beta)$ field. Typically polarizabilities are measured in Compton scattering processes. Pions cannot be used as a fixed target, but they can be scattered off the Coulomb potential of a heavy nucleus. In the reaction $\pi^{-} \gamma \rightarrow \pi^{-} \gamma$ the polarizabilities appear as a correction factor to the Born cross section of a point-like particle. A measurement of the cross section for this process provides access to this factor, and therefore to the polarizabilities.

COMPASS has taken data where this reaction is embedded into the scattering of a pion beam off a Nickel target. A large energy deposit in the electromagnetic calorimeter is required to select the photon in the final state, the photon in the initial state is ensured by removing hadronic interactions with a cut on low photon virtuality $Q^{2}$. Photons in the final state are required to have a ratio $x_{\gamma}$ of the photon and the initial beam energy between 0.4 and 0.9 , in the kinematic region of stable trigger efficiency and muon identification. Due to limited statistics in the region of phase space sensitive to $\alpha_{\pi}+\beta_{\pi}$ (at lower $x_{\gamma}$ ), the assumption of $\alpha_{\pi}=-\beta_{\pi}$ has to be made. In this case the polarizability 


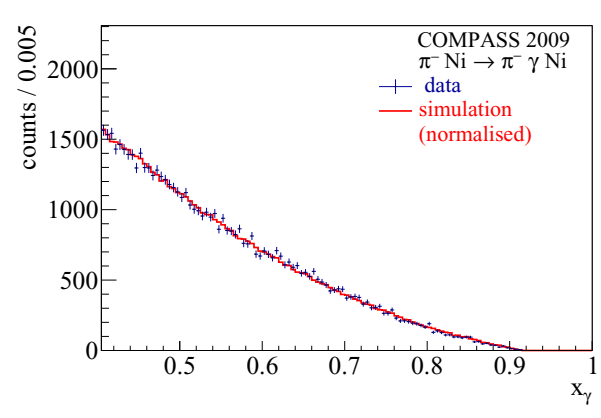

(a) comparison of measurement and simulation

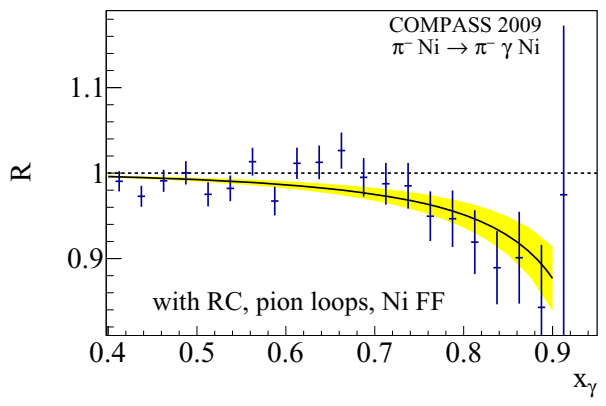

(b) ratio $R$

Figure 8. Number of measured and simulated events, and their ratio $R$ used to extract the polarizabilities.

should manifest itself in a decreasing ratio $R$ of the measured cross section over the simulated cross section for a point-like particle towards higher $x_{\gamma}$ (see figure 8(b), see [6] for more details on the data selection and the way the polarizability is obtained). A value for the polarizability of $\alpha_{\pi}=$ $(2.0 \pm 0.6 \pm 0.7) \cdot 10^{-4} \mathrm{fm}^{3}$ is extracted using this approach. A control measurement with a muon beam is performed, yielding a false polarizability of the muon that is compatible with zero. The observed pion polarizability is at variance from previous experiments, but in agreement with the prediction from chiral perturbation theory [7].

\section{Outlook}

COMPASS has collected a large data set that is used to perform spectroscopy in the light-meson sector. Apart from the results presented here, also new insights concerning the spin-exotic candidate $\pi_{1}$ (1600) are gained, not only in the two $(3 \pi)^{-}$channels discussed above, but also in the $\pi^{-} \eta$ and $\pi^{-} \eta^{\prime}$ channels. Finally, the analysis of $(5 \pi)^{-}$and $\pi^{-} \eta \eta$ final states should provide access towards higher masses.

In 2012 a considerably larger data set has been taken in order to improve on the measurement of the pion polarizabilities. The range in $x_{\gamma}$ was extended, providing a handle also on $\alpha_{\pi}+\beta_{\pi}$. For the first time, a measurement of the kaon polarisabilites is foreseen.

\section{References}

[1] COMPASS collaboration, P. Abbon et al., Nucl. Instrum. Methods Phys. Res., Sect. A 577, 455 (2007).

[2] COMPASS collaboration, M.G. Alekseev et al., Phys. Rev. Lett. 104, 241803 (2010).

[3] A. Austregesilo on behalf of the COMPASS Collaboratian, PoS(Hadron 2013)102.

[4] COMPASS collaboration, C. Adolph et al., European Physical Journal A 50, 79 (2014).

[5] J.L. Rosner, Phys. Rev. D 23, 1127 (1981).

[6] S. Huber on behalf of the COMPASS Collaboration, PoS(Hadron 2013)125.

[7] J. Gasser, M.A. Ivanov, and M.E. Sainio, Nucl. Phys. B 745, 84 (2006). 
